\title{
LAS UCRONÍAS EN LA LITERATURA ARGENTINA
}

\author{
POR \\ Luis Pestarini \\ Biblioteca Nacional, Argentina
}

La especulación sobre lo que hubiera sucedido si determinado acontecimiento histórico no se hubiese producido o lo hubiera hecho de un modo diferente, está presente en las ciencias sociales desde fines del siglo XIX, como señala Pablo Capanna ("La nariz..."37-38). Habitualmente se considera que el libro que da origen de manera formal a la especulación contrafáctica histórica es la antología If It Had Happened Otherwise: Lapses into Imaginary History (1931), preparada por J. C. Squire. Este volumen incluye ensayos de personalidades reconocidas como Winston Churchill, quien especula sobre lo que hubiese pasado si el General Lee no hubiera ganado la batalla de Gettysburg, o G. K. Chesterton, que se atreve a reflexionar en lo que hubiera sucedido si Don Juan de Austria se hubiese casado con María, Reina de Escocia. ${ }^{1}$ Como se puede apreciar, para generar un escenario ucrónico o una historia alternativa, lo primero que hace el autor es seleccionar el punto de inflexión o divergencia y trazar, a partir de allí, un devenir histórico razonable. El primer ejemplo al que se suele recurrir cuando se quiere clarificar este concepto es un universo en el que el Eje ganó la Segunda Guerra Mundial, pero son innumerables los puntos críticos desde donde se puede "cambiar" la historia: la Armada Invencible conquista Inglaterra y la Revolución Industrial no se produce, el Sur gana la Guerra de Secesión y Estados Unidos (los estados del norte) se convierten en una nación subdesarrollada, la Peste Negra extermina casi toda la población de Europa, de modo que la Civilización se desarrolla en China, América indígena, el Mundo Árabe y la India. Estos escenarios fueron utilizados por escritores anglosajones que frecuentan la ciencia ficción para desarrollar historias en ambientes ucrónicos.

No todos los críticos concuerdan con que el relato ucrónico es una de las formas de las ciencia ficción. Matthew Schneider-Mayerson afirma: "Some scholars have considered the alternate history to be a subgenre within science fiction, but this is misleading, since

\footnotetext{
María Estuardo, probablemente la más conocida de los monarcas de Escocia, era católica, lo que hubiera
} llevado a que Inglaterra también fuera predominantemente católica. 
it can also take the form of a traditional novel or a work of fantasy. Although this article is concemed with literary counterfactuals, a number of films and television shows have engaged in counterfactual history, and one can argue that the increasingly popular video games with historical settings that allow for allohistorical developments should be placed in the same category" (65). Si bien en la literatura contemporánea se han dado muchas combinaciones entre los géneros editoriales, la mayoría de las ucronías literarias se desarrollan con cierto rigor a partir del punto de divergencia, un rigor que coincide con el de la ciencia ficción "dura" 2 que toma una premisa -la telepatía, por ejemplo-y desarrolla el relato estrictamente a partir de aceptar esto. El mayor o menor éxito o verosimilitud de la historia tiene que ver más con la capacidad del autor que con las limitaciones de este subgénero de la ciencia ficción. Capanna se expresa en esta dirección: "Pese a que, como hemos visto, esta problemática es bastante antigua y surgió de las especulaciones filosóficas o de la reflexión de los historiadores, la ciencia ficción (incluyendo sus provincias literarias aledañas) se apoderó del tema una vez que descubrió sus posibilidades narrativas" ("La nariz..." 42). Por último, si hace falta establecer algún tipo de vínculo directo con la ciencia ficción, hay uno incontrastable: la mayoría de las ucronías fueron y son escritas por autores que habitualmente adscriben a la ciencia ficción.

Aunque aparentemente sencilla de definir, las historias alternativas o ucronías presentan algunas dificultades al intentar fijar sus límites. ¿Podemos considerar como ucronía un relato de ambiente contemporáneo con una logia secreta de vampiros? ¿Y una historia ambientada en un mundo indeterminado, que podría ser el futuro o uno imaginario? Para los fines de este artículo, consideraremos como historia alternativa o ucronía a aquellos relatos cuyo punto de divergencia, o sus consecuencias, son explícitas.

Ahora bien, por su alcance, por la utilización de elementos de otros géneros, incluso por su objetivo político, hay diferentes tipos de ucronías. Expongamos algunos ejemplos de historias alternativas para aclarar esto. En 1945, Alemania y Estados Unidos no llegan a la guerra, la Unión Soviética no sobrevive al conflicto como una única nación, y en consecuencia la Guerra Fría se da entre las dos naciones mencionadas inicialmente. Por lo descripto aquí, este mundo alternativo podría servir de escenario para una historia con las características de un thriller pero cobra otra dimensión al conocer a sus autores: William R. Forstchen, un escritor de ciencia ficción irrelevante, y Newt Gingrich, el entonces líder republicano del Congreso. La novela es un líbelo político.

Más allá de las posibles motivaciones de una obra literaria, ya sean morales, de propaganda o de entretenimiento, hay una forma de clasificar las ucronías vinculada a

\footnotetext{
2 Se denomina ciencia ficción dura a aquella que está desarrollada con fuerte validación de los conocimientos científicos de la época, basándose, en particular, en ciencias como la química, la física o la astronomía. Durante los últimos veinte años estas etiquetas se han desdibujado hasta casi desaparecer pero nos permitimos su uso para agilizar la explicación.
} 
elecciones narrativas. Las ucronías más recurrentes son aquellas en las que se plantea un punto de divergencia como los que hemos mencionado y la acción transcurre en algún momento futuro de ese punto. A veces se detalla el encadenamiento de cambios, en ocasiones es invisible mientras el relato sea consistente. A veces ni siquiera se hace explícito el punto de divergencia, sólo se sospecha. Podemos denominar a este tipo de ucronía como "pura".

Ahora bien, si hay ucronías puras debe haber una contraparte, que podemos llamar "mixta": si en el primer tipo tenemos un único universo, en el segundo la narración nos lleva a más de uno, originados a partir de distintos cursos de acción en el punto de divergencia. El ejemplo más reiterado de este tipo de ucronía es El hombre en el castillo, de Philip K. Dick, donde como un espejo al mundo en el que suceden las acciones, hay otro en una novela que circula clandestinamente, La langosta se ha posado, en la cual el Eje perdió la Segunda Guerra, pero ese universo tampoco es el nuestro. Es poco sabido, pero Dick escribió algunos capítulos de una continuación de El hombre en el castillo, que se centraban en un viaje experimental al universo alternativo descripto por La langosta se ha posado.

En nuestra caracterización, tenemos un tercer y último tipo de ucronía, que es aquella que se centra en narrar el suceso que se convierte en el punto de divergencia y no necesariamente los sucesos que desencadena. Incluso puede ser que este punto de divergencia se corrija para reencausarse en el devenir "real".

En la literatura argentina hay ejemplos de los tres tipos de ucronías que, aunque dispersos y de diversa calidad narrativa, establecen una leve tradición de más de seis décadas. En algunos casos, el punto de divergencia altera el mundo entero, en otros son sólo cambios locales. En las páginas siguientes vamos a hacer un repaso de algunas de las principales ucronías de la literatura argentina, dejando de lado textos que, si bien bordean los elementos de la historia alternativa, no lo hacen estrictamente desde la narrativa, como Ucronías argentinas (2009), de Javier Aguirre, Eduardo Blanco y Fernando Sánchez, una compilación de textos que parten de hechos contrafácticos con el único objetivo de generar derivaciones absurdas y humorísticas (entre las que cabe mencionar lo que sucede cuando los cardenales eligen a un argentino para suceder a Juan Pablo II). Tampoco serán considerados ensayos de historia contrafáctica, como el de Juan Carlos Torre, aparecido en Ensayos sobre movimiento obrero y peronismo, en el que especula con un 17 de octubre sin el peronismo, ni las novelas vinculadas especialmente con una coyuntura política como El día que mataron a Alfonsín (1986) y El día que mataron a Cafiero (1987), de Dalmiro Sáenz y Sergio Joselovsky.

El relato ucrónico argentino más antiguo conocido es el magistral "La trama celeste" (1948), de Adolfo Bioy Casares. La fecha de publicación cobra relevancia dado que 
según el sitio Uchronia, ${ }^{3}$ que funciona como principal referente en el tema, la ucronía moderna más antigua es Lest Darkness Fall, de L. Sprague De Camp, publicada menos de una década antes, en 1939. El relato de Bioy Casares narra una situación inexplicable que sufre el protagonista, el Capitán Ireneo Morris, quien, en un vuelo de prueba, sufre un accidente para despertar varios días después en un hospital. No lo reconocen sus compañeros de armas y se debate si no será un espía al que hay que ejecutar. Escapa y luego de algunas aventuras vuelve a volar para abandonar ese mundo. Bioy Casares emplea un recurso ingenioso para resaltar los diferentes mundos: algunos nombres de calles son distintos. Morris descubre que Gales no existió en aquel mundo, y por ende él, descendiente de galeses, tampoco. El narrador de "La trama celeste" relata una historia que le contó otra persona, dándola por cierta. Cierra el texto un comentario de un tercero que desacredita la historia y su justificación en la Teoría de la Pluralidad de los Mundos. No se hace explícito el punto de divergencia, probablemente ni siquiera lo haya fijado el autor. Sabemos solamente que Gales no existe y que Cartago sobrevivió al devastador ataque musulmán de comienzos del siglo VIII.

Otro ejemplo de lo que llamamos "ucronía mixta" podría ser "De navegantes", uno de los capítulos unitarios de Trafalgar (1979), de Angélica Gorodischer. El narrador, Trafalgar Medrano, es un comerciante que realiza viajes a otros mundos y relata sus aventuras a sus interlocutores en un café de Rosario. El condicional del comienzo del párrafo tiene una explicación: Trafalgar no circula de un universo a otro sino que arriba a un planeta que es idéntico al nuestro, poco antes del descubrimiento de América. Su intervención modifica la conquista de América y la torna más pacífica, aunque al final del relato se pregunta si hizo lo correcto, porque en América no se dará una potencia capaz de enfrentar a la Alemania de Hitler. Gorodischer pinta con trazos gruesos los rasgos de esta ucronía sin preocuparse por hacerla verosímil. De hecho, esta es una de las características de las historias de Trafalgar.

Las "ucronías puras" son más abundantes en la literatura argentina y, en general, están trabajadas con más detalle. Tal es el caso de Buenos Ayres City (1968), de Marcos Victoria, intelectual de nombre en las décadas de los cincuentas y sesentas, amigo de Borges, que prologó el segundo libro de la editorial Minotauro, ${ }^{4}$ Más que humano, de Theodore Sturgeon. El punto de divergencia es que fue exitosa la Segunda Invasión Inglesa realizada sobre Buenos Aires en 1807. ${ }^{5}$ Las Provincias Unidas son una colonia

3 Uchronia es una importante fuente de referencias, especial pero no exclusivamente a relatos en inglés. También es de interés Historia alternativa (http://es.althistory.wikia.com), destinado a desarrollar lo que indica su nombre, pero sin el sesgo literario.

4 Minotauro, vale la pena recordarlo, ejerció la función de legitimar a la ciencia ficción en el ambiente intelectual argentino y latinoamericano, con una selección de títulos, ediciones y traducciones muy cuidada.

5 Entre 1806 y 1807, Inglaterra intentó ocupar ambas orillas del Río de la Plata. En 1807 un ejército de más de 10.000 hombres fue derrotado en Buenos Aires, y precipitó la caída de la ocupación de Montevideo. 
inglesa, ordenada, con restos culturales españoles - especialmente en el interior del paíspero donde se cumple la fantasía de cierta clase media de la segunda mitad del siglo XX: es un estado a la altura de Canadá o Australia. Narrada desde el punto de vista de un periodista estadounidense que viene a hacer una nota sobre la situación real de las Provincias Unidas, desfilan personajes como el Puma, un político populista tenebroso (Perón) o el Gran Inca, un líder indígena que reivindica las culturas y tradiciones nativas y es tratado como un alienado. Los únicos argentinos que pelean contra los ingleses se encuentran en las Islas Malvinas y son un grupo desorganizado, que viste andrajos y no suscita ninguna simpatía. Victoria, amigo de Borges, transmite a través de Buenos Ayres City, de un modo muy crudo, la visión de la sociedad liberal burguesa antiperonista de los años cincuentas y sesentas, sin intención paródica o crítica.

El peronismo -y Perón mismo-no han estado ausentes en las ucronías. En el relato "El oro de Tiresias" (1991), de Gorodischer, Perón no es liberado en 1945 y nunca llega a presidente, muriendo en la cárcel. Narrada por un estudiante de mediados del siglo XXI que investiga el periodo, la historia prosigue con una sucesión de gobiernos militares. En “1953” (2008), Alejandro Alonso narra un íntimo encuentro entre Ernesto Sábato y Perón que fuerza este último con la excusa de presentar su pésame por el fallecimiento de la esposa y los hijos de Sábato en un accidente automovilístico. Perón termina confesando a Sábato que, en realidad, fue para invitarlo a integrarse a unos equipos de investigación que trabajan secretamente sobre una nave espacial extraterrestre que se recuperó del océano. Alonso trata con mucho cuidado a ambos personajes, aunque su simpatía está con Sábato. "1957" (2011) está ambientada en el futuro de "1953", en el cual Perón ha muerto. Es un relato policial ambientado en una ucronía de la que no se ofrecen muchos detalles. Ambos relatos son parte de una novela inconclusa y, en principio, se puede afirmar que el punto de divergencia es la aparición de la nave extraterrestre. En "1957" se menciona que Perón murió en 1955.

En clave política pero satírica debe leerse Un guión para Artkino (2008), de Fogwill. Escrita a fines de la década del setenta, su autor extravió el manuscrito por más de dos décadas. Transcurre en 1994, cuando Argentina es parte de la Unión de Repúblicas Socialistas Soviéticas, que domina casi todo el mundo. Al protagonista, un Fogwill de 54 años, le encargan la escritura del guión de una película para realizarse en el Hollywood de entonces, Artkino, ${ }^{6}$ en Moscú. El texto desliza algunas ironías deliciosas, como la mención de un homenaje al camarada Borges, autor de la novela Horas proletarias y de la nouvelle "Mañanitas metalúrgicas", y emplea un dispositivo que aparece con cierta frecuencia en otras ucronías: el cruzamiento con otro texto ficticio que sirve para fortalecer la idea de la ucronía. Ya lo hemos visto en El hombre en el castillo de Philip K. Dick, con La langosta se ha posado. Otro título clásico de

Artkino fue el nombre de la distribuidora de cine soviético en América entre 1940 y 1982. 
la literatura ucrónica, El sueño de hierro, de Norman Spinrad, es una novela dentro de otra novela: en la primera, Hitler emigró a Estados Unidos y se convirtió en escritor de ciencia ficción; la segunda es el texto íntegro de una de sus novelas, El señor de la esvástica. En el libro de Fogwill cumple ese rol el propio guión por el que fue contratado y que está ambientado enel año 2018, cuando el comunismo tiene el dominio completo del mundo. Un guión para Artkino es una sátira de la burocracia cultural comunista, y no está exento de guiños personales.

Argentina ocupada por los nazis también dio lugar a una ucronía pura: "La noche de al lado" (1985), de Sergio Gaut vel Hartman. El autor ha publicado varios relatos ucrónicos, algunos de ellos microcuentos, y durante un poco más de un año condujo, junto al español Antonio Álamo, la sección Uficción en la publicación electrónica Axxón, donde se incluían relatos ucrónicos, aunque la mayoría de ellos no son de autores argentinos. En "La noche de al lado", el nazismo domina Argentina -y se intuye que gran parte del mundo-, y la trama deviene en un thriller en el cual se busca reemplazar a Hitler, quien acaba de regresar de un viaje a Alfa Centauri, con un clon suyo. Como en otros relatos ucrónicos de este autor, el caso de "El destino no es ciego" (2004), donde un viajero extranjero evita las muertes prematuras de Gardel y Horacio Quiroga, y los reúne con Perón, Gaut vel Hartman parece disfrutar especialmente el placer de ser un demiurgo cambiando la historia de personajes importantes, pero no avanza en la dirección de la especulación ni en el de la sátira, por lo que los elementos que configuran una ucronía no se profundizan.

Tal vez el relato ucrónico más elaborado como tal sea ;Argentinos, a vencer! (2012), de Juan Simeran, en el cual Argentina no perdió la Guerra de las Malvinas. Narrado treinta años después del triunfo, cuando todavía se combate esporádicamente en las islas, a través de una historia de amores despechados e intrigas, se describe detalladamente una Argentina aislada del mundo, atrasada tecnológicamente, en crisis económica continua, y un clima opresivo y represivo como el del peor momento de la dictadura militar que se inició en 1976. Previsiblemente, el gobierno militar salió fortalecido de la contienda y extendió su dominio hasta entrado el siglo XXI. Se impone un nacionalismo superficial de enaltecimiento a lo marcial que esconde un profundo estado de corrupción y descomposición social. Simeran recupera un recurso que empleó Bioy Casares en "La trama celeste" para resaltar las diferencias con nuestro mundo: las denominaciones de las calles repiten, quizás en exceso, los nombres de militares, muchos ligados con la dictadura de Videla. Como ya hemos señalado en otro ejemplo, para dar mayor verosimilitud a la historia, al finalizar muchos capítulos se recurre a textos de un libro escolar que idolatran al Conscripto Sosa como un ejemplo para los escolares, o a fragmentos del periódico El caudillo. ;Argentinos, a vencer! propone que, si el desenlace de la Guerra de las Malvinas hubiera sido otro, los gobiernos militares se hubieran extendido largamente en el tiempo y Argentina hubiera sufrido el aislamiento internacional, la represión y la decadencia social más grave de su historia. 
No podemos concluir el repaso de algunas ucronías literarias de las que hemos dado en llamar puras sin mencionar una que tiene más que ver con una fantasía del argentino medio que con especulación contrafáctica: en "No, gracias"(2004), de Hernán Domínguez Nimo, Diego Maradona no se hace adicto a las drogas en 1982, la Selección Argentina de Fútbol gana varios mundiales gracias a su presencia (y no sólo el de 1986), abandona el Barcelona luego de varias temporadas exitosas para recalar en el Juventus (no en el Nápoles), donde se convierte en ídolo. Luego de dejar el fútbol practica varios deportes con éxito desparejo para volver a la Selección de Fútbol y ganar otra Copa Mundial.

Nos queda una última categoría de ucronías para la cual encontrar ejemplos en la literatura argentina, aquellas que se centran en el punto de divergencia. Tanto la obra teatral Los indios estaban cabreros (1958), de Agustín Cuzzani, como la novela El conquistador (2006), de Federico Andahazi, se centran en una misma idea: antes de la llegada de los españoles a América arriba un grupo de aztecas a Europa, quienes siguen un periplo que los regresará a México. Hay algunas coincidencias puntuales que bien pueden ser fruto de desarrollar una misma idea disparadora. Los descendientes de Cuzzani iniciaron acciones legales por plagio, acciones que fueron desestimadas en los juzgados. ${ }^{7}$

La obra de Cuzzani es una sátira que sigue los pasos de tres aztecas (Tupa, Tonatio y Teuche) quellegan a España, huyendo porque han fracasado varios intentos revolucionarios en contra de su régimen sangriento, y son atrapados por pescadores en sus redes, que los consideran pescados por sus curiosos rasgos. Estamos en 1491. Son encarcelados pero finalmente llegan hasta Isabel la Católica, que está bajo el acoso de Cristóbal Colón para que le brinde apoyo para cruzar el océano. El final es amargo: Tupa, el líder de los aztecas que habían sido condenados a muerte antes del encuentro con la reina, afirma: "No iré con los tuyos, Reina Isabel. Esos barcos llevan otras cosas además de sabios y matemáticos. Llevan la muerte y la esclavitud y la guerra a lo más profundo de las comarcas que encuentren. Yo he decidido la suerte del futuro, pero morirán muchos hombres... ¡Y yo quiero ser el primero en morir!" (54). Tal vez Cuzzani no fuera consciente de había escrito una ucronía, pero está claro que Los indios estaban cabreros lo es. El texto, gracias a su condición teatral, facilita algunas humoradas ingeniosas: un policía detiene a Colón en la calle y le pregunta su nombre: "Ah... la cosa no es muy clara", responde. "Me llaman Cristóbal Colón, o Xhristopherens Columbus, o Colombo, o Colomo, o Colom, o..." (50).

El conquistador, ganadora del Premio Planeta argentino, tiene mucho menos humor y presta especial atención a la cultura azteca, al punto en que casi la mitad del libro se desarrolla antes del viaje del protagonista, Quetza, un sabio aventurero que está obligado a demostrar que la tierra es redonda o de lo contrario se ejecutará una condena a muerte. Con ojos de extranjero llega a Huelva y lo primero que presencia es una quema de la Inquisición. Las visiones religiosas de ambos pueblos, el azteca y el

\footnotetext{
$<$ http://axxon.com.ar/not/186/c-1861100.htm>.
} 
europeo, son contrastadas: en ambas hay sacrificios humanos, por ejemplo, y los cleros tienen enorme poder político. Pero Andahazi no se queda en una comparación tan directa aunque no puede eludir la conclusión de que el pensamiento religioso es irracional y contrario al científico (Quetza es el equivalente azteca a un Da Vinci). Una vez que la comitiva azteca recorrió Europa, parte hacia oriente, a China y Japón, para cruzar el Pacífico en sus naves con pruebas de la existencia de otros continentes como animales e inventos. Poco antes de arribar a sus costas una tormenta hunde los barcos y sólo alcanzan tierra algunos pocos náufragos, entre ellos Quetza, pero sin ninguna de las pruebas que recogieron. Nadie cree sus advertencias de que deben conquistar Europa antes que los europeos lleguen a sus tierras, y termina sus días esperando la llegada de las naves enemigas.

Hay varias coincidencias entre ambos textos, coincidencias que en cierto sentido son inevitables: los intentos de los protagonistas por cambiar el futuro son inútiles, o tal vez debamos decir que en la obra de Cuzzani lo que hace es acelerar los acontecimientos, pero en ambos libros, a pesar de la divergencia con la historia "real", esta se corrige. Ambos finales son amargos, no hay forma de evitar la tragedia que se derramará sobre América. Otra coincidencia es que los protagonistas de ambos libros (Tupa y Quetza), tienen un encuentro breve con Colón, tentación que ninguno de los dos autores pudo evitar. Sin embargo, hay una visión muy distinta sobre los pueblos americanos: el texto de Cuzzani asume que los europeos están mil años adelantados a los indios mientras que en el de Andahazi, autor contemporáneo, se habla de dos civilizaciones que han evolucionado de manera distinta, con sus propias fortalezas y flaquezas. ${ }^{8}$

Un último ejemplo de historia alternativa sobre el punto de divergencia puede ser "La noche reina" (1996), unos de los cuentos ganadores del Premio FAIGA, del autor de este artículo. Ambientado en los tiempos inmediatos posteriores a la Revolución de Mayo de 1810, narra el viaje de Mariano Moreno hacia Inglaterra para solicitar el reconocimiento de la nueva nación y buscar colaboración. El narrador es un viajero temporal que viene de un futuro donde América Latina unida es una gran potencia y está estudiando a uno de los forjadores de esa línea temporal. Confirma la sospecha de que el comandante del barco está envenenando a Moreno hasta que finalmente, a pesar de su intento de evitarlo, muere. Se reitera, como los textos de Cuzzani y Andahazi, una constante en las ucronías de este tipo: tienden a neutralizarse luego del punto de divergencia o a generar el mundo "real" a partir de este punto."

8 Para un análisis más profundo de El conquistador, se recomienda Pintarle bigote a la Mona Lisa: las ucronías, de Campo Ricardo Burgos López, único libro íntegramente dedicado a analizar las ucronías escrito en español. El libro de Andahazi es la única ucronía argentina trabajada en el de Burgos López.

9 Sobre la finalización de la preparación de este artículo nos encontramos con otra ucronía sobre el Descubrimiento de América que no queremos dejar de mencionar: "Las crónicas de la serpiente emplumada", conformada por El libro del mensajero (2009), El libro del guerrero (2010), El libro del 
Hasta aquí hemos intentado una relación de varios relatos ucrónicos de la literatura argentina, relación que no resulta tan sencilla porque, por un lado, las ediciones de los libros casi nunca se identifican como ucronías, y no pocas veces los autores no comprenden que están incursionando en esta categoría (Argentinos ; a vencer! y Buenos Ayres City reconocen su pertenencia), y, por otro, no existe bibliografía sobre el tema. Es seguro que hay muchos textos que han evadido la búsqueda, tanto largos como cortos, de modo que este trabajo puede ser considerado sólo como provisorio.

En esta relación constituimos tres categorías de ucronías con el único objetivo de establecer cierto ordenamiento, una tipificación básica, pero su pueden admitir otras. Las categorías que se establecieron son las "ucronías puras", donde el relato se desarrolla en un mundo alternativo sin intervención de otras dimensiones, las "ucronías mixtas", donde están presentes más de un universo alternativo, y las "ucronías sobre el punto de divergencia", que describen las acciones y hechos históricos que son modificados pero no necesariamente sus consecuencias.

En ocasiones, las ucronías son verdaderas declaraciones de posturas políticas e ideológicas, a veces de manera no intencional. Pero es riesgoso calificar las posiciones de los autores a partir de sus obras de ficción, aunque en ciertas oportunidades son muy manifiestas. La ucronía es, como buena parte de la mejor ciencia ficción, narrativa especulativa que se afianza en un cambio conceptual -en este caso un punto de divergencia- para reflexionar sobre sus consecuencias sobre el hombre y la sociedad.

\section{BiBLIOGRAFÍA}

Alonso, Alejandro. “1953”. Cuásar 46, marzo 2008: 1-9.

"1957”. Próxima 9, marzo 2011: 16-22.

Andahazi, Federico. El conquistador. Buenos Aires: Planeta, 2006.

Burgos López, Campo Ricardo. Las ucronías: pintarle bigote a la Mona Lisa. Bogotá:

Universidad Sergio Arboleda, 2009.

Capanna, Pablo. Ciencia ficción: utopía y mercado. Buenos Aires: Cántaro, 2007.

"La nariz de Cleopatra y el teniente Bonaparte". El Péndulo 12 (1986): 36-53.

Córdoba Cornejo, Antonio. ¿Extranjero en tierra extraña?: el género de la ciencia ficción en América Latina. Sevilla: Universidad, 2011.

Cuzzani, Agustín. Una libra de carne. Los indios estaban cabreros. Buenos Aires: Talia, 1958.

Domínguez Nimo, Hernán. “No, gracias”. Axxón 141 (ago. 2004)

heredero (2010) y Regreso al principio (2012), de Edgardo Civallero. En esta serie de novelas, no se sabe más nada de Colón después de su partida y en 1521 llega a la costa europea la armada azteca, invirtiendo el signo de la conquista. Los textos íntegros se pueden leer en http://cronicasdelaserpienteemplumada. blogspot.com.ar/ 
Fogwill, Rodolfo Enrique. Un guión para Artkino. Buenos Aires: Mansalva, 2008.

Gallagher, Catherine. "When Did the Confederate States of America Free the Slaves?" Representations 98/1 (Spring 2007): 53-61.

Gaut vel Hartman, Sergio. "El destino no es ciego". Axxón 135 (feb. 2004)

"La noche de al lado". Sinergia 10 (verano 1985): 85-122.

Gorodischer, Angélica. "De navegantes". Trafalgar (1979): 61-87.

"El oro de Tiresias". Axxón 22 (Jul 1991): 12-33.

Piatti, Barbara; Hurni, Lorenz. "Mapping the Ontologically Unreal: Counterfactual Space in Literature and Cartography". The Cartographic Journal 46:4 (nov. 2009):333-342.

Pestarini, Luis. "La noche reina". Al sur del tiempo (1996): 61-73.

Schneider-Mayerson, Matthew. "What Almost Was: The Politics of the Contemporary Alternate History Novel." American Studies with American Studies International 50/3-4 (Fall-Winter 2009): 63-83.

Simeran, Julio. Argentinos ... ; a vencer! Buenos Aires: Fan, 2012.

Victoria, Marcos. Buenos Ayres City. Buenos Aires: Losada, 1968.

Winthrop-Young, Geoffrey. "The Third Reich in Alternate History: Aspects of a GenreSpecific Depiction of Nazi Culture." Journal of Popular Culture 39/5 (oct. 2006): 878-896. 